\title{
Color and spatial feature for content-based image retrieval
}

\author{
Mohan S. Kankanhalli ${ }^{\text {a,*}}, 1$, Babu M. Mehtre ${ }^{\text {b,2 }}$, Hock Yiung Huang ${ }^{c}$ \\ ${ }^{a}$ Institute of Systems Science, National University of Singapore, Kent Ridge, Singapore 119597, Singapore \\ b Image Processing and New Technologies Group, CMC Center, Gachibowli, Hyderabad 500019, India \\ ${ }^{\mathrm{c}}$ School of Electrical and Electronic Engineering, Nanyang Technological University, Nanyang Avenue, Singapore 639798, Singapore
}

Received 22 April 1997

\begin{abstract}
Most of the currently available image database systems provide a text-based retrieval function called keyword retrieval, where users specify 'keywords' such as titles, attributes, and categories of themes. But many times it is not easy for users to specify suitable keywords for a particular retrieval. Besides, building a large image database with complete description of contents is a very difficult task. In this paper, we present a content-based retrieval method which obviates the need to describe certain contents of an image to be archived and retrieved. The proposed method computes image features automatically from a given image and they can be used to archive and/or retrieve images. These features are based on color and its spatial distribution information in an image. We have also developed a similarity measure to compare the color and spatial feature similarity of two images. This technique has been developed and tested for content-based similarity retrieval of images on two databases consisting of: (i) 100 test images and (ii) 800 actual trademarks images. The experimental results show a high efficiency of retrieval. (c) 1999 Published by Elsevier Science B.V. All rights reserved.
\end{abstract}

Keywords: Color; Spatial feature; Similarity retrieval; Distance; Content-based

\section{Introduction}

Traditional database systems provide sophisticated and efficient techniques that handle alphanumeric data very well. When it comes to the storage and retrieval of visual data (images, photographs, drawings, paintings) they employ description of images for the retrieval of desired

\footnotetext{
* Corresponding author.

${ }^{1}$ Supported by the Real World Computing Partnership, Japan.

${ }^{2}$ This work was performed at the Institute of Systems Science.
}

images. The process of building an image database which employs description of images for archival and retrieval is time consuming, subjective, and error prone. Automatic characterization of images and eliminating the need to provide manual description of images is getting wider attention of the research community (Wu et al., 1995; Picard, 1995). This is a new and expanding area of research called content-based retrieval of image and multimedia data. Wokimoto et al. (1993) have presented a drawing image database system which helps similarity retrieval of logically structured drawings. They employ a graph representation for storage and retrieval of drawings. Rickham and Stonham (1993) have proposed a neural network 
based coding scheme for retrieval of images according to the degree of similarity with a query image. Hibler et al. (1992) have proposed an augmented entity relationship model to overcome the limitations of traditional key word system. Picard and Minka (1995) have used texture features to automatically extract the description of regions in an image.

An image can be characterized by color, shape, texture, and spatial features. Similarity retrieval of images using only color (Kankanhalli et al., 1996; Babu et al., 1995; Stricker and Orengo, 1995) and only shape (Wu et al., 1995) has been discussed in the literature. In this paper, we describe a technique for similarity retrieval of images using color and spatial features. This method is useful for queries like "give me all images where there is a red ball somewhere in the upper right corner among other things". The query could be also posed as a retrieval of similar images for a given example image. To test the efficacy of retrieval we use the efficiency of retrieval as a figure of merit. The efficiency of retrieval (Kankanhalli et al., 1996), $\eta_{T}$, for a given short-list $T$ (the top $T$ most similar images) is given by

$\eta_{T}= \begin{cases}\frac{n}{N} & \text { if } N \leqslant T, \\ \frac{n}{T} & \text { if } N>T,\end{cases}$

where $n$ is the number of similar images retrieved and $N$ is the total number of similar images in the database.

A lot of work has been done in image retrieval based on the color feature. Since this material has been covered elsewhere (Kankanhalli et al., 1996; Stricker and Orengo, 1995; Swain and Ballard, 1991), we will not review it here. To the best of our knowledge, there have been only two attempts to combine color and spatial information (Gong et al., 1994; Hsu et al., 1995). In the Gong et al. method (Gong et al., 1994), the authors divide the image into a $3 \times 3$ grid having 9 regions and then compute the histogram for each of these regions as well as the whole image. Then they use the histogram intersection method (Swain and Ballard, 1991) to match corresponding regions of images. Their method has two drawbacks - firstly, it is not adaptive to the image, i.e. if an object straddles across multiple regions, then the perfor- mance will degrade severely. Secondly, histogram matching has been found not to be a very good feature for capturing color content of images (Babu et al., 1995; Stricker and Orengo, 1995). Hsu et al. (1995) propose a method which selects a set of $N$ representative colors $(N / 2$ for background and $N / 2$ for object), analyzes the spatial information of these $N$ colors using a maximum entropy discretization method and finally integrates both these information to compute the similarity measure by using a variation of the histogram intersection technique. While it is adaptive for spatial regions, the disadvantage of this method is that the choice of $N / 2$ colors each for the background and the object is not adaptive. Also, object is defined to be the small window in the center of the image which may not be always necessarily true. In addition, the limitations of the histogram matching method also apply here. In contrast to these two approaches, our method uses the object distribution information of the image at hand to adaptively partition the color objects and hence does not impose any arbitrary subdivision of the image. Moreover, we do not use the histogram matching technique for the similarity measure computation.

The paper is organized as follows. Color and spatial feature is introduced in Section 2. The similarity measure is described in Section 3 followed by test results and discussion in Section 4 . The conclusions and future work is given in Section 5 and references are given at the end.

\section{Color and spatial feature}

The pixels of a color image can be regarded as points in the 3-D color space. Their coordinates in the 3-D color space are the color values of the pixels. Usually the number of objects in an image is small, hence the reflection characteristics of each object in natural light tends to be consistent and it results as clusters in the color space. If clustering is performed in the 3-D color space, a few clusters will be obtained, each cluster corresponds to one of the dominant colors in the image. A representative sample (e.g. the mean color) of all such clusters can be used to define a color feature 
representing the color information of the image (Kankanhalli et al., 1996). The spatial location (e.g. centroid of a color region in image coordinates) and the population of the clusters in the image define another feature, representing the spatial distribution information of the color image. These two features can thus be used to capture the color content of the image along with the spatial distribution of the colors in the image.

\subsection{The clustering algorithm}

The input images are represented as RGB primary color images. Color clustering can be used to find the color clusters and assign a representative color to each of these clusters. From the color clusters, spatial clustering can be used to separate each color cluster into a number of smaller spatial clusters. Fig. 1 gives an example of color clusters and spatial clusters in an image.

\subsubsection{Color clustering}

The color clustering algorithm (based on (Kankanhalli et al., 1996)) presented here is an unsupervised method that assumes each color cluster follows a normal distribution with one peak and there is sufficient distance between every two color clusters. The color clustering algorithm is as follows:

1. Obtain the RGB components of image.

2. Find all color clusters.

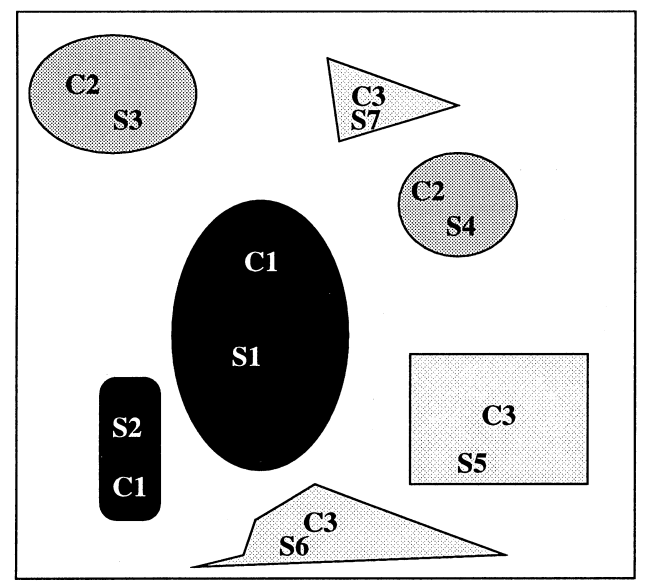

2.1. Compute the color distance of each pixel from the existing color clusters. If no color clusters exist, then a new color cluster is created. The color distance is given by $\sqrt{(\Delta R)^{2}+(\Delta G)^{2}+(\Delta B)^{2}}$.

2.2. If the minimum color distance is less than the minimum threshold value, then a match is found. Otherwise, a new color cluster is created. We have used a minimum threshold of 50 for a $400 \times 400$ image which is equal to $0.03125 \%$ of the total number of pixels. An order of magnitude variation of this parameter does not change the retrieval results.

2.3. For each match, the RGB values and the population of the clusters are updated. The new representative color of the cluster is the weighted average of the original cluster and the current pixel's color.

3. Sort the clusters in a descending order, based on the cluster population.

4. Determine the number of clusters which do not have a very small population. We have considered a cluster with $\leqslant 1 \%$ population as small. A variation of a factor of 5 in this threshold did not affect the retrieval results.

5. If the number of clusters found in step 4 is less than in step 2, merge the very small clusters to their nearest color clusters. The nearest cluster

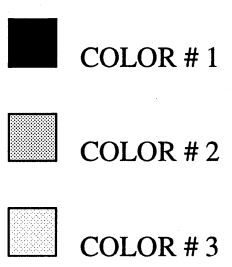

NUMBER OF COLOR CLUSTERS $=3(\mathrm{C} 1, \mathrm{C} 2, \mathrm{C} 3)$

NUMBER OF SPATIAL CLUSTERS $=7 \quad(\mathrm{~S} 1, \mathrm{~S} 2, \ldots, \mathrm{S} 7)$

Fig. 1. Example of an image with 3 colors and 7 spatial clusters. 
is computed based on the color distance metric. The representative for this color cluster is then the weighted mean of the two original clusters.

6. For each pixel, compute the color distance to different cluster. Assign the pixel to the cluster for which the color distance is minimum. Thus every pixel gets assigned to one cluster.

Thus, after this algorithm is applied on an input image, the color clusters will be obtained and each pixel of the image will be assigned to one of these clusters. Note that spatial correlation information can be used to improve the results of the clustering using methods based on Markov random fields (Young and $\mathrm{Fu}, 1986$ ). Moreover, alternate color clustering methods such as (Celenk, 1988; Andreadis et al., 1990; Healey, 1992; Ebi et al., 1994) could also be used. A better clustering method will only improve the retrieval results.

\subsubsection{Spatial clustering}

Using the output generated by the color clustering algorithm, the following steps are used to obtain the spatial clusters:

1. Split the image into different color layers. The number of color layers is equal to the number of color clusters that is determined by the color clustering algorithm.

2. Do a connected components labeling to separate the spatial clusters (Nadler and Smith, 1993).

3. For each color layer, sort the spatial clusters in a descending order, based on the cluster population.

4. Determine the number of clusters which do not have a very small population.

5. Clusters which have population less than the lower threshold is discarded. We used a lower threshold of 50 pixels $(0.03125 \%)$.

6. For clusters which have population less than the upper threshold (we used an upper threshold of 1000 pixels $=0.625 \%$ ) but more than the lower threshold, a density function is computed as follows:

- Assume that points $\left(x_{1}, y_{1}\right)$ and $\left(x_{2}, y_{2}\right)$ are the corner points of a rectangle which bounds a particular spatial cluster. Find the maximum length $l_{\max }$ of the box.

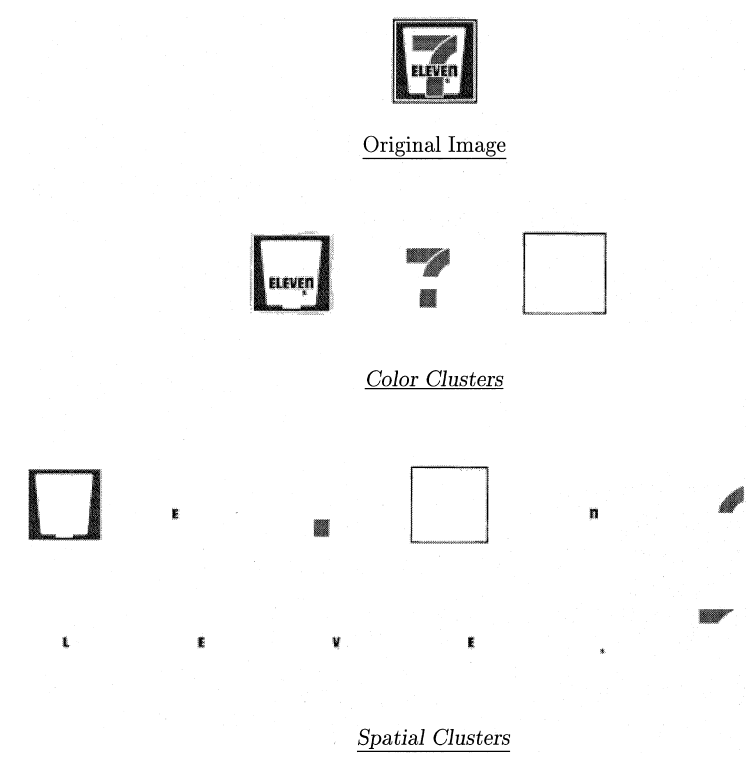

Fig. 2. Test results for color and spatial clustering algorithm.

$l_{\max }=\max \left(\left\|x_{2}-x_{1}\right\|,\left\|y_{2}-y_{1}\right\|\right)$.

- The density function of the cluster is given as

density, $\rho=\frac{\text { population of cluster }}{\left(l_{\max }\right)^{2}}$

o if the density, $\rho \geqslant 10 \%$ then the cluster is retained. Otherwise the cluster is ignored. The advantage of this method is that it will eliminate those clusters which have a low density, e.g. a thin line that forms a cluster. This is particularly useful in eliminating noise which manifests in the form of short lines. This type of noise is introduced at object boundaries due to the image scanning process.

Fig. 2 shows sample results of color clustering and spatial clustering. It can be seen that the algorithm separates the color clusters and spatial clusters of the test image.

\subsection{The color and spatial feature vector}

Suppose an image $I$ of size $N$ pixels has $m$ color clusters and $n$ spatial clusters, with $n \geqslant m$. Each color clusters $C_{c, i}$ is defined as follows:

$C_{c, i}=\left\{R_{i}, G_{i}, B_{i}, \lambda_{c, i}, x_{c, i}, y_{c, i}\right\}, \quad i=1,2, \ldots, m$, 
where $\left(R_{i}, G_{i}, B_{i}\right)$ is the representative color of the color cluster (mean) and $\lambda_{c, i}$ is the fraction of the pixels in that color cluster as compared to the total number of pixels.

$\lambda_{c, i}=\frac{\text { Number of pixels } \in C_{c, i}}{N}$,

$\left(x_{c, i}, y_{c, i}\right)$ is the centroid (in image coordinates) of the $i$ th color cluster. The color cluster feature of image $I$ is defined as

$\bar{f}_{c}=\left\{C_{c, i} \mid i=1,2, \ldots, m\right\}$.

Each color-spatial cluster is defined as follows:

$C_{s, i}=\left\{R_{i}, G_{i}, B_{i}, \lambda_{s, i}, x_{s, i}, y_{s, i}\right\}, \quad i=1,2, \ldots, n$,

where $\left(R_{i}, G_{i}, B_{i}\right)$ is the representative color of the color-spatial cluster and $\lambda_{s, i}$ is the fraction of the pixels in that color-spatial cluster as compared to the total number of pixels.

$\lambda_{s, i}=\frac{\text { Number of pixels } \in C_{s, i}}{N}$,

$\left(x_{s, i}, y_{s, i}\right)$ is the centroid of the $i$ th color-spatial cluster. The color-spatial cluster feature of image $I$ is then defined as

$\bar{f}_{s}=\left\{C_{s, i} \mid i=1,2, \ldots, n\right\}$.

\section{The similarity measure}

In this section, we describe the computation of the similarity measure between a pair of images. The similarity measure presented here compares the color information of two images, as well as the spatial distribution of the colors. Since it is not always true that the two images to be compared have similar or the same number of color and spatial clusters, the measure will penalize any missing color or spatial clusters. This corresponds to the intuitive notion of image similarity. The similarity computation includes finding the closest cluster for each of the color and color-spatial clusters and then calculating the distance measure.

\subsection{Finding the closest cluster}

Assume that the query image $Q$ has $m$ colour clusters and $p$ spatial clusters and the database image $I$ has $n$ color clusters and $q$ spatial clusters.
The closest color cluster assignment function $P_{c}$ maps every color cluster $i$ of image $Q$ to the closest color cluster $P_{c}(i)$ of image $I$. This is a function in the sense that it is a 1-to-1 assignment. The computation of the closest color cluster assignment function, $P_{c}$ is as follows:

1. Form the distance matrix, $D_{m n}$,

$$
D_{m n}=\left[d_{m n}\right]_{m \times n},
$$

where $d_{m n}$ is the color distance between $C_{m}^{Q}$ and $C_{n}^{I}$.

2. Find the minimum entry $(a, b)$ in matrix $D_{m n}$ and note that $P_{c}(a)=b$.

3. Strike off row $a$ and column $b$ of the matrix $D_{m n}$. If the matrix is non-degenerate, go to step 2 else stop. A matrix is considered degenerate when the number of rows or columns which is not yet struck off is zero.

For every matched color cluster, a spatial match analogous to the computation of $P_{c}$ is done, using the spatial centroid for distance measure,

$d=\sqrt{\left(x_{s_{p}}-x_{s_{q}}\right)^{2}+\left(y_{s_{p}}-y_{s_{q}}\right)^{2}}$.

This will produce a closest color-spatial cluster assignment function, $P_{s}$, which maps every colorspatial clusters of image $Q$ to the closest one in $I$.

\subsection{Similarity measure for color and spatial feature}

Suppose that the query image $Q$ has $m$ color clusters and $p$ spatial clusters and the database image $I$ has $n$ colour clusters and $q$ spatial clusters. Also the closest color cluster assignment function, $P_{c}$, maps $k$ color clusters of $Q$ to $I$ and the closest color-spatial cluster assignment function, $P_{s}$, maps $l$ color-spatial clusters of $Q$ to $I$. For computing the similarity, the distance measure, $D(Q, I)$, is given by

$D(Q, I)=\omega_{1} \Psi_{1}+\omega_{2} \Psi_{2}+\omega_{3} \Psi_{3}+\omega_{4} \Psi_{4}+\omega_{5} \Psi_{5}$,

where

$$
\begin{aligned}
& \Psi_{1}=\frac{\sum_{i=1}^{\max (m, n)} \operatorname{cdist}\left(C_{i}^{Q}, c_{P_{c}(i)}^{I}\right)}{k}, \\
& \Psi_{2}=m \sum_{i=1}^{\max (m, n)} \sqrt{\left(\lambda_{c, i}^{Q}-\lambda_{c, P_{c}(i)}^{I}\right)^{2}},
\end{aligned}
$$


$\Psi_{3}$

$=\frac{\sum_{i=1}^{\max (m, n)} \sqrt{\left(x_{c, i}^{Q}-x_{c, P_{c}(i)}^{I}\right)^{2}+\left(y_{c, i}^{Q}-y_{c, P_{c}(i)}^{I}\right)^{2}}}{k(m: 2+m \% 2)}$,

$\Psi_{4}=\frac{\sum_{i=1}^{\max (p, q)} \sqrt{\left(\lambda_{s, i}^{Q}-\lambda_{s, P_{s}(i)}^{I}\right)^{2}}}{m}$,

$\Psi_{5}$

$$
=\frac{\sum_{i=1}^{\max (p, q)} \sqrt{\left(x_{s, i}^{Q}-x_{s, P_{s}(i)}^{I}\right)^{2}+\left(y, s_{i}^{Q}-y_{s, P_{s}(i)}^{I}\right)^{2}}}{m l},
$$

where $\omega_{1}, \omega_{2}, \omega_{3}, \omega_{4}, \omega_{5}$ are the weight factors, and $\operatorname{cdist}\left(C_{i}, C_{P_{c}(i)}\right)$

$$
= \begin{cases}\operatorname{coldis}\left(C_{i}^{Q}, C_{P_{c}(i)}^{I}\right) & \text { if } \lambda_{c, i}^{Q}, \lambda_{c, P_{c}(i)}^{I}>0, \\ 0 & \text { otherwise. }\end{cases}
$$

The distance formula has five components:

1. $\Psi_{1}$ is the color distance between the color clusters. This measures the proximity of the colors in the given pair of color images.

2. $\Psi_{2}$ is the relative frequency of pixels of the corresponding color clusters. Given that two images have similar colors, then this factor takes into account the relative proportions of the colors in the two images for ranking. It also helps to rank appropriately when the database image and query image have different number of colors. It prefers the images in the database that have same number of colors as in the query image, and penalizes otherwise.

3. $\Psi_{3}$ is the spatial distance between the color clusters. This allows those database images to come on top which have spatially closer clusters to the clusters in the query image and penalizes other database images which do not satisfy this criterion.

4. $\Psi_{4}$ is the relative frequency of pixels of the corresponding color-spatial clusters. This factor takes into account same size and number of spatial clusters in both the query and database images. The images satisfying this criteria appear at the top end of the retrieved image and others are pushed down the list.

5. $\Psi_{5}$ is the spatial distance between the color-spatial clusters. It helps to brings out all those images in the database that have spatial clusters close to the spatial clusters in the query image.
The five components, $\Psi_{1}, \Psi_{2}, \Psi_{3}, \Psi_{4}$ and $\Psi_{5}$ in the similarity measure are weighted by factors, $\omega_{1}, \omega_{2}, \omega_{3}, \omega_{4}$ and $\omega_{5}$, respectively. Thus different aspects of the similarity measure can be emphasized by increasing the weight factors. For example, if the color similarity between a pair of corresponding color clusters is considered the most important criterion for matching, then the weight factor, $\omega_{1}$, can be increased accordingly to emphasize its importance. Similarly the weight factors, $\omega_{3}$ and $\omega_{5}$, can be increased accordingly to emphasize the importance of the location of the color and spatial clusters in the distance measure. $\omega_{2}$ and $\omega_{4}$ can also be increased to emphasize the importance of the relative population of the corresponding clusters. The appropriate settings of $\omega_{1}, \omega_{2}, \omega_{3}, \omega_{4}$ and $\omega_{5}$ depends on the overall importance of each of the aspects in determining image similarity for the application under consideration. In our experiments, we used $\omega_{1}=$ $10.0, \omega_{2}=1.0, \omega_{3}=10.0, \omega_{4}=2.5$ and $\omega_{5}=5.0$. These values were determined by a domain expert's (trademark officer) input for obtaining the (expert's) desired ranking of images. The input consisted of the relative weights of the similarity measure components. It was then experimentally confirmed, using the synthetic image database, that the desired ranking was indeed obtained using these weights.

\section{Test results and discussion}

The content based retrieval scheme using color and spatial feature has been implemented on a SparcStation 10, using C language. We have built two database consisting of (a) 100 synthetic test images and (b) 800 Trademarks images.

Fig. 3 shows the retrieval results for a query image for a 100-images database with a short list of size $T=10$. The query image consists of two color objects, red rectangle surrounded by a blue ball. From the output of similarity retrieval, it is clear that all the images are similar to the query image, and also they are ranked in the order of decreasing similarity. This test on synthetic images was carried out for an illustrative purpose prior to the test on the trade mark images. 


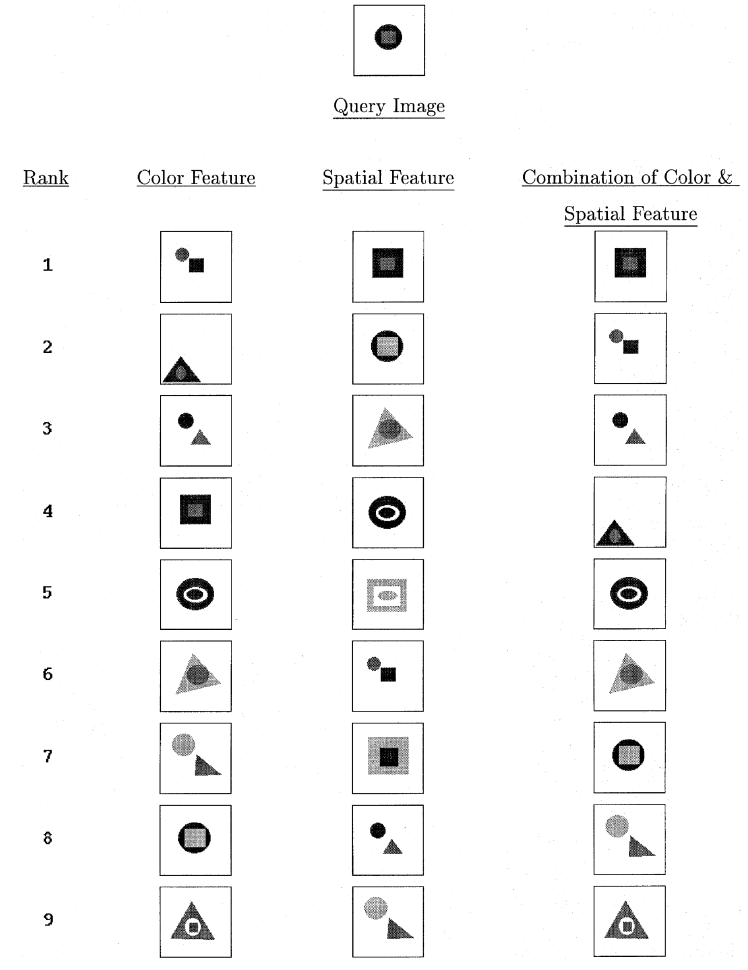

Fig. 3. Sample results of images retrieved using color feature, spatial feature, and combined color and spatial feature.

Fig. 4 shows the retrieval results for an actual trademark image for an 800-images trademarks image database. For trademark registration, it is required to ensure that the submitted trademark does not conflict with any of the already registered (existing) trademarks. The query image is a red symbol adjacent to a black string somewhere in the centre of the image. Here, the retrieved output consists of all trademarks that have red and black components in the centre. (Note that we consider only the color and spatial feature here. We do not perform textual string comparison of the trademark, which can be easily done using existing methods.)

To illustrate the efficacy of our method, we have presented the retrieved results using (a) only color, (b) only spatial feature and (c) color and spatial feature combined, in three separate columns. The images are ranked from most similar to least similar in a top-to-bottom scan order, in each column.
To evaluate the technique rigorously we have carried out tests which were designed as follows: 10 queries (images) which represent the population well were picked. They were representative in the sense that they covered almost all the colors as well as types of spatial distributions occurring in the 800 -image database used. For each query image, a list of similar images found in the database is first manually determined. For image retrieval, the enduser is a human being. So the "similarity" of any system should correspond to the human perceptual similarity. In our case, we have used a particular class of images - "trademark images". We therefore used the judgement of a trademark officer for deciding similarity. The officer provided the desired ranked output from the database for each of the ten query images. The color and spatial feature of the query image is then compared to the database features to obtain a short list of similar images. Then the efficiency of retrieval $\left(\eta_{T}\right)$ was calculated. Table 1 shows the retrieval efficiency for a set of 10 representative queries, giving individual and average figures. Note that $N$ in the table shows the number of images in the database which are similar to the query image (determined by the domain expert). The retrieval efficiency of only the color feature, only spatial feature and the combination of both the features are presented in Table 1.

From Table 1, it can be seen that the similarity measure (Eq. (1)) gives a high efficiency of retrieval, $\eta_{T}>90 \%$. For example, for a short list size of $T=10$, the average retrieval efficiency is $95.1 \%$. This shows that this technique has the ability to rank images, taking into account both the color and spatial distribution features. While we have performed the experiments on the trademark images, our technique will work for any class of images which have at least some dominant colors and pixels of these colors possess spatial contiguity.

\section{Conclusions}

We have described a color and spatial feature based image retrieval technique for content based image retrieval. The proposed technique has been tested on two separate databases consisting of 100 
6fitflox

Query Image

$\underline{\text { Rank }}$ Color Feature Spatial Feature Combination of Color \&

$\underline{\text { Spatial Feature }}$

1

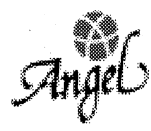

Groutera

ind

2

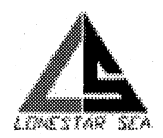

S ruwrr

S rowaxy

3

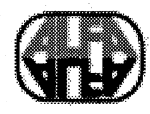

⿶Clop

Rroutera

4

AUNW

monren

axu

5

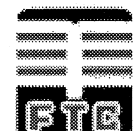

- $-6 r a 44$

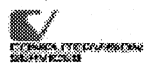

6

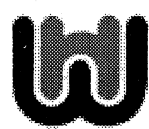

aASF

MITCEFES

7

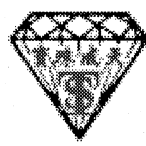

LQLonus

- Exoquip

8

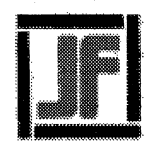

๒กก

a BASF

9

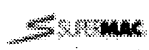

STEMER

signtiks

Fig. 4. Sample results of images retrieved using color feature, spatial feature and combination of color and spatial feature. 
Table 1

Retrieval efficiency, $\eta_{T}$, using similarity measure based on color feature, spatial feature, and combination of both color and spatial features ( $N$ is the number of similar images in the database for the corresponding query image)

\begin{tabular}{|c|c|c|c|c|c|c|c|c|c|c|c|c|}
\hline \multirow[b]{2}{*}{ Query image } & \multicolumn{4}{|c|}{ Color feature } & \multicolumn{4}{|c|}{ Spatial distribution } & \multicolumn{4}{|c|}{ Combination of both } \\
\hline & $N$ & $T=5$ & $T=10$ & $T=20$ & $N$ & $T=5$ & $T=10$ & $T=20$ & $N$ & $T=5$ & $T=10$ & $T=20$ \\
\hline 1 & 100 & 1.00 & 1.00 & 1.00 & 27 & 1.00 & 1.00 & 0.75 & 12 & 1.00 & 0.90 & 0.75 \\
\hline 2 & 140 & 1.00 & 1.00 & 1.00 & 35 & 1.00 & 0.90 & 0.90 & 20 & 1.00 & 1.00 & 0.80 \\
\hline 3 & 17 & 1.00 & 0.80 & 0.88 & 15 & 1.00 & 0.80 & 0.87 & 13 & 1.00 & 0.90 & 1.00 \\
\hline 4 & 28 & 1.00 & 1.00 & 0.90 & 20 & 1.00 & 0.80 & 0.75 & 4 & 1.00 & 1.00 & 1.00 \\
\hline 5 & 140 & 1.00 & 1.00 & 0.95 & 13 & 0.80 & 0.70 & 0.92 & 4 & 1.00 & 1.00 & 1.00 \\
\hline 6 & 140 & 1.00 & 1.00 & 1.00 & 13 & 1.00 & 0.80 & 0.85 & 6 & 0.83 & 1.00 & 1.00 \\
\hline 7 & 7 & 1.00 & 0.86 & 0.86 & 3 & 0.33 & 0.33 & 0.33 & 6 & 1.00 & 1.00 & 1.00 \\
\hline 8 & 4 & 1.00 & 1.00 & 1.00 & 15 & 1.00 & 0.70 & 0.87 & 3 & 1.00 & 1.00 & 1.00 \\
\hline 9 & 6 & 0.60 & 0.67 & 0.67 & 31 & 0.60 & 0.60 & 0.70 & 3 & 0.67 & 1.00 & 1.00 \\
\hline 10 & 12 & 0.80 & 0.70 & 0.75 & 15 & 0.80 & 0.80 & 0.80 & 7 & 0.57 & 0.71 & 0.86 \\
\hline Average & & 0.940 & 0.903 & 0.901 & & 0.853 & 0.743 & 0.774 & & 0.907 & 0.951 & 0.941 \\
\hline
\end{tabular}

synthetic images and about 800 real trademark images. The technique performs very well and gave very good retrieval efficiency, $\eta_{T}>90 \%$ for both the databases. The retrieval output based on this technique gives images that are ranked in the order of similarity, taking into account both the color and the spatial features. In the future we plan to work on an indexing mechanism for efficient retrieval of images and avoid sequential matching of all image (features) in the database.

\section{Acknowledgements}

We thank our colleague A. Desai Narasimhalu for suggesting the problem.

\section{References}

Andreadis, I., Browne, M.A., Swift, J.A., 1990. Image pixel classification by chromaticity analysis. Pattern Recognition Letters. 11, 51-58.

Babu, G.P., Mehtre, B.M., Kankanhalli, M.S., 1995. Color indexing for efficient image retrieval. Multimedia Tools Appl. 1 (4), 327-348.

Celenk, M., 1988. A recursive clustering technique for color picture segmentation. In: Proc. IEEE Conference on Computer Vision and Pattern Recognition - CVPR 1988. pp. 437-444.

Ebi, N., Lauterbach, B., Anheier, W., 1994. An image analysis systems for automatic data acquisition from colored scanned maps. Machine Vision Appl. 7, 148-164.
Gong, Y., Zhang, H., Chuan, H.C., Sakauchi, M., 1994. An image database system with content capturing and fast image indexing abilities. In: Proc. IEEE International Conference on Multimedia Computing and Systems. Boston, pp. 121-130.

Healey, G., 1992. Segmenting images using normalized color. IEEE Trans. Systems, Man, and Cybernetics 22 (1), 64-73.

Hibler, J.N.D., Leung, C.H., Mannock, K.L., 1992. System for content-based storage and retrieval in an image database. In: Proc. SPIE Conference on Image Storage and Retrieval Systems. SPIE Vol. 1662, pp. 80-92.

Hsu, W., Chua, T.S., Pung, H.K., 1995. An integrated colorspatial approach to content-based image retrieval. In: Proceedings of the Third ACM International Multimedia Conference. San Francisco.

Kankanhalli, M.S., Mehtre, B.M., Wu, J.K., 1996. Clusterbased color matching for image retrieval. Pattern Recognition 29 (4), 701-708.

Nadler, M., Smith, E.P., 1993. Pattern Recognition Engineering. Wiley, New York, pp. 165-167.

Picard, R.W., 1995. Light-years from Lena: Video and image libraries of the future. MIT Media Laboratory Perceptual Computing Section Technical Report No. 339.

Picard, R.W., Minka, T.P., 1995. Vision texture for annotation. ACM Multimedia Systems 3 (1), 3-14.

Rickham, R., Stonham, J., 1993. Similarity retrieval from image databases - Neural Networks can deliver. In: Proc. SPIE Conference on Storage and Retrieval for Image and Video Databases. SPIE Vol. 1908, pp. 85-94.

Stricker, M., Orengo, M., 1995. Similarity of color images. In: Proc. SPIE Conference on Storage and Retrieval for Image and Video Databases III. SPIE Vol. 2420, pp. 381-392.

Swain, M.J., Ballard, D.H., 1991. Color indexing. Internat. J. Comput. Vision 7 (1), 11-32.

Wokimoto, K., Shima, M., Tanaka, S., Maeda, A., 1993. Content-based retrieval applied to drawing image data- 
bases. In: Proc. SPIE Conference on Storage and Retrieval for Image and Video Databases. SPIE Vol. 1908, pp. 74-84. Wu, J.K., Narasimhalu, A.D., Mehtre, B.M., Lam, C.P., Gao, Y.J., 1995. CORE: A content based retrieval engine for multimedia information systems. ACM Multimedia Systems 3 (1), 25-41.

Young, T.Y., Fu, K.S. (Eds.), 1986. Handbook of Pattern Recognition and Image Processing. Academic Press, New York. 